Article

\title{
$g$-Expectation for Conformable Backward Stochastic Differential Equations
}

\author{
Mei Luo ${ }^{1}$, Michal Fečkan ${ }^{2,3}{ }^{\mathbb{D}}$, Jin-Rong Wang ${ }^{1, *}$ and Donal O'Regan ${ }^{4}$ \\ 1 Department of Mathematics, Guizhou University, Guiyang 550025, China; meiluomath@163.com \\ 2 Department of Mathematical Analysis and Numerical Mathematics, Comenius University in Bratislava, \\ Mlynská dolina, 84248 Bratislava, Slovakia; michal.feckan@fmph.uniba.sk \\ 3 Mathematical Institute of Slovak Academy of Sciences, Štefánikova 49, 81473 Bratislava, Slovakia \\ 4 School of Mathematical and Statistical Sciences, National University of Ireland, 999014 Galway, Ireland; \\ donal.oregan@nuigalway.ie \\ * Correspondence: jrwang@gzu.edu.cn
}

\begin{abstract}
In this paper, we study the applications of conformable backward stochastic differential equations driven by Brownian motion and compensated random measure in nonlinear expectation. From the comparison theorem, we introduce the concept of $g$-expectation and give related properties of $g$-expectation. In addition, we find that the properties of conformable backward stochastic differential equations can be deduced from the properties of the generator $g$. Finally, we extend the nonlinear Doob-Meyer decomposition theorem to more general cases.
\end{abstract}

Keywords: nonlinear expectation; $g$-expectation; Doob-Meyer decomposition theorem

MSC: 26A33

check for

updates

Citation: Luo, M.; Fečkan, M.; Wang, J.-R.; O'Regan, D. g-Expectation for Conformable Backward Stochastic Differential Equations. Axioms 2022, 11, 75. https://doi.org/10.3390/ axioms11020075

Academic Editor: Delfim F. M. Torres

Received: 21 December 2021

Accepted: 10 February 2022

Published: 14 February 2022

Publisher's Note: MDPI stays neutral with regard to jurisdictional claims in published maps and institutional affiliations.

Copyright: (C) 2022 by the authors. Licensee MDPI, Basel, Switzerland. This article is an open access article distributed under the terms and conditions of the Creative Commons Attribution (CC BY) license (https:// creativecommons.org/licenses/by/ $4.0 /)$.

\section{Introduction}

The initial research motivation of nonlinear expectations came from risk measurement and option pricing in financial applications. The Allais paradox, Ellsberg paradox and Simon's "bounded rationality" theory, and so forth, all show that decision-making in reality is contrary to the hypothesis of expected utility theory. Economists have found that the linearity of classical mathematical expectation (that is, the additivity of probability measures) is the main reason for this kind of problem so researchers wanted to find a new tool which can not only retain some properties of classical mathematical expectations, but also solve financial problems with highly dynamic and complex characteristics.

In the 1950s, Choquet [1] extended the Lebesgue integral to non-additive measure and obtained the Choquet expectation. However, this nonlinear expectation does not have dynamic compatibility and is not suitable for solving practical financial problems. In 1997, Peng [2] introduced a new nonlinear expectation, namely the $g$-expectation, based on the backward stochastic differential equation driven by Brownian motion. The $g$-expectation retains all the basic properties of the classical expectation except linearity [3], and it can be applied to the dynamic risk measurement of actuarial and financial valuation. Subsequently, Royer [4] studied the backward stochastic differential equation driven by Brownian motion and Poisson random measure, and introduced the corresponding g-expectation and a large number of studies show that this $g$-expectation can be applied to financial problems (see [5-9]). Recently, Long et al. [10] proposed a multi-step scheme on time-space grids for solving backward stochastic differential equations, and Chen and Ye [11] investigated solutions of backward stochastic differential equations in the framework of Riemannian manifold. From the paper [12], we could get the averaging principle for backward stochastic differential equations and the solutions can be approximated by the solutions to averaged stochastic systems in the sense of mean square under some appropriate assumptions. 
In addition, coupled forward backward stochastic differential equations driven by the G-Brownian motion were studied in [13], while [14] investigated the solvability of fully coupled forward-backward stochastic differential equations with irregular coefficients.

The above papers concern research on integer order derivative, while the works of conformable type derivative are very few ([15-20]). The conformable derivative not only has some properties of fractional derivative, but also some properties of integer order derivatives. We discussed the necessity of studying conformable backward stochastic differential equations in [21]. In the present paper, we study g-expectation for conformable backward stochastic differential equations.

This paper is mainly divided into four parts. In the second section, we give some definitions and theorems. In the third section, we study the relationship between $g$-expectation and the filtered consensus expectation, and we give some properties of $g$-expectation. We find that the $g$-expectation can be considered as a nonlinear extension of the Girsanov transformation. In the final section, we prove the Doob-Meyer decomposition theorem under mild assumptions.

\section{Preliminaries}

Let $B(\cdot)$ be a standard Brownian motion defined on the complete probability space $(\Omega, \mathcal{F}, \mathbb{P})$ with the filtration $\left\{\mathcal{F}_{t}\right\}_{0 \leq t \leq T}$ satisfying the usual hypotheses of completeness and right continuity. $\mathfrak{B}(\mathbb{R})$ denotes the Borel sets of $\mathbb{R}$ and $\mathbb{E}$ denotes the expected value. A stochastic process $V(\omega, t)$ is a real function defined on $\Omega \times[0, T]$ such that $\omega \rightarrow V(\omega, t)$ is $\mathcal{F}$-measurable for any $t \in[0, T]$. A stochastic process $V$ is called $\mathcal{F}_{t}$-adapted if $\omega \rightarrow V(\omega, t)$ is $\mathcal{F}_{t}$-measurable for any $t \in[0, T]$. The natural filtration is completed with sets of measure zero. By $\mathcal{P}$ we denote the $\sigma$-field. A process $V: \Omega \times[0, T] \rightarrow \mathbb{R}$ is called $\mathcal{F}$-predictable if it is $\mathcal{F}$-adapted and $\mathcal{P}$-measurable. A process is called càdlàg if its trajectories are rightcontinuous and have left limits. The term a.s. means almost surely with respect to the probability measure. Inspired by [22], we define some spaces that we will use:

$L_{Q}^{2}(\mathbb{R})=\left\{\right.$ measurable function $\varphi: \mathbb{R} \rightarrow \mathbb{R} ; \int_{\mathbb{R}}|\varphi(s)|^{2} Q(d s)<\infty ; Q$ is a $\sigma$ - finite measure $\}$,

$\mathbb{L}^{2}\left(\Omega, \mathcal{F}_{T}, \mathbb{P}\right)=\left\{\mathcal{F}_{T}\right.$ - measurable random variable $\left.\xi: \Omega \rightarrow \mathcal{F}_{T} ; \mathbb{E}\left[|\zeta|^{2}\right]<\infty\right\}$,

$\mathbb{H}^{2}(\mathbb{R})=\left\{\right.$ predictable process $\left.Y: \Omega \times[a, T] \rightarrow \mathbb{R} ; \mathbb{E}\left[\int_{a}^{T}|Y(t)|^{2} d t\right]<\infty\right\}$,

$\mathbb{H}_{N}^{2}(\mathbb{R})=\left\{\right.$ predictable process $\left.Z: \Omega \times[a, T] \times \mathbb{R} \rightarrow \mathbb{R} ; \mathbb{E}\left[\int_{a}^{T} \int_{\mathbb{R}}|Z(t, s)|^{2} Q(t, d s) \eta(t) d t\right]<\infty\right\}$,

$\mathbb{S}^{2}(\mathbb{R})=\left\{\right.$ adapted, càdlàg process $\left.X: \Omega \times[a, T] \rightarrow \mathbb{R} ; \mathbb{E}\left[\sup _{t \in[a, T]}|X(t)|^{2}\right]<\infty\right\}$,

$\mathbb{L}^{2}\left(\Omega, \mathcal{F}_{t}, \mathbb{P}\right)=\left\{\mathcal{F}_{t}-\right.$ measurable random variable $\left.\left.\xi: \Omega \rightarrow \mathcal{F}_{t} ; \mathbb{E}\left[|\xi|^{2}\right]<\infty\right]\right\}$.

Furthermore, for any constant $\sigma$, we introduce the norms of spaces $\mathbb{H}^{2}, \mathbb{H}_{N}^{2}$ and $\mathbb{S}^{2}$ as:

$$
\begin{aligned}
& \|Y\|_{\mathbb{H}^{2}}^{2}=\mathbb{E}\left[\int_{a}^{T} e^{\sigma t}|Y(t)|^{2} d t\right], \\
& \|Z\|_{\mathbb{H}_{N}^{2}}^{2}=\mathbb{E}\left[\int_{a}^{T} \int_{\mathbb{R}} e^{\sigma t}|Z(t, s)|^{2} Q(t, d s) \eta(t) d t\right], \\
& \|X\|_{\mathbb{S}^{2}}^{2}=\mathbb{E}\left[\sup _{t \in[a, T]} e^{\sigma t}|X(t)|^{2}\right] .
\end{aligned}
$$

Definition 1. (see [2] (Definition 3.1)) A functional $\mathcal{E}: \mathbb{L}^{2}\left(\Omega, \mathcal{F}_{T}, P\right) \rightarrow \mathbb{R}$ is called a nonlinear expectation if it satisfies the following properties:

(i) Strict monotonicity: if $X_{1} \geq X_{2}$ a.s., $\mathcal{E}\left[X_{1}\right] \geq \mathcal{E}\left[X_{2}\right]$, and if $X_{1} \geq X_{2}$ a.s., $\mathcal{E}\left[X_{1}\right]=\mathcal{E}\left[X_{2}\right] \Leftrightarrow$ $X_{1}=X_{2}$ a.s.

(ii) preserving of constants: $\mathcal{E}[c]=c$, for any constant $c$. 
Definition 2. (see [2] (Definition 3.2)) A nonlinear expectation $\mathcal{E}$ is a filtration consistent expectation $\left(\mathcal{F}\right.$-consistent expectation) if for any $\zeta \in \mathbb{L}^{2}\left(\Omega, \mathcal{F}_{T}, \mathbb{P}\right)$ and $a \leq t \leq T$, there exists a random variable $\xi \in \mathbb{L}^{2}\left(\Omega, \mathcal{F}_{t}, \mathbb{P}\right)$ such that $\mathcal{E}\left[\zeta 1_{A}\right]=\mathcal{E}\left[\xi 1_{A}\right], \forall A \in \mathcal{F}_{t}$, where $\xi$ is uniquely defined. We denote $\xi=\mathcal{E}\left[\zeta \mid \mathcal{F}_{t}\right]$, which is called the conditional expectation of $\zeta$ with respect to $\mathcal{F}_{t}$. Therefore, we can write it as $\mathcal{E}\left[\zeta 1_{A}\right]=\mathcal{E}\left[\mathcal{E}\left[\zeta \mid \mathcal{F}_{t}\right] 1_{A}\right], \forall A \in \mathcal{F}_{t}$.

Lemma 1. (see [4](Lemma A.1)) Let $A(\cdot)$ be an increasing predictable process. We consider its decomposition as a sum of a continuous and a purely discontinuous process: $A(t)=A^{1}(t)+A^{2}(t)$. We also consider a càdlàg martingale $W(\cdot)$, bounded in $\mathbb{L}^{2}$.

(i) For any stopping time $\tau$ such that $a \leq \tau \leq T$,

$$
\mathbb{E}\left[\int_{a}^{\tau} \triangle W(s) d A^{1}(s)\right]=0
$$

(ii) For any predictable stopping time $\tau$ such that $a \leq \tau \leq T$,

$$
\mathbb{E}\left[\int_{a}^{\tau} \triangle W(s) d A^{2}(s)\right]=\mathbb{E}\left[\sum_{a \leq s \leq \tau} \triangle W(s) \triangle A^{2}(s)\right] .
$$

Lemma 2. (see [21] (Theorem 3.5)) Suppose $U(\cdot)=U(X(\cdot), \cdot) \in C^{2,1}\left(\mathbb{R} \times \mathbb{R}_{+}, \mathbb{R}\right)$. Then, for any $a \leq t \leq T$, we have

$$
\begin{aligned}
D_{\rho}^{a} U(t) d \frac{(t-a)^{\rho}}{\rho}= & \left(\frac{\partial u}{\partial t}-(t-a)^{\rho-1} g(t, X(t), Y(t), Z(t, \cdot)) \frac{\partial u}{\partial x}\right. \\
& \left.+\frac{1}{2}(t-a)^{2(\rho-1)} Y^{2}(t) \frac{\partial^{2} u}{\partial x^{2}}\right) d t+(t-a)^{\rho-1} Y(t) \frac{\partial u}{\partial x} d B(t) \\
& +(t-a)^{\rho-1} \frac{\partial u}{\partial x} \int_{\mathbb{R}} Z(t, s) \tilde{N}(d t, d s) \\
& +\frac{1}{2}(t-a)^{2(\rho-1)} \frac{\partial^{2} u}{\partial x^{2}} \int_{\mathbb{R}} Z^{2}(t, s) N(d t, d s), 0<\rho \leq 1 .
\end{aligned}
$$

Lemma 3. (see [22] (Theorem 2.5.1)) Let B be a $(\mathbb{P}, \mathcal{F})$-Brownian motion, $N$ be a $(\mathbb{P}, \mathcal{F})$-random measure with compensator $\vartheta(d \tau, d s)=Q(\tau, d s) \eta(\tau) d \tau$. Assume an equivalent probability measure $\mathbb{Q} \sim \mathbb{P}$ with a positive $\mathcal{F}$-martingale:

$$
\frac{d M(t)}{M(t)}=\phi(t) d B(t)+\int_{\mathbb{R}} \kappa(t, s) \tilde{N}(d t, d s), M(a)=1,
$$

where $\phi(\cdot)$ and $\kappa(\cdot, \cdot)$ are the $\mathcal{F}$-predictable processes satisfying

$$
\begin{aligned}
& \int_{a}^{T}|\phi(t)|^{2} d t<\infty, \int_{a}^{T} \int_{\mathbb{R}}|\kappa(t, s)|^{2} Q(t, d s) \eta(t) d t<\infty, \\
& \kappa(t, s)>-1, a \leq t \leq T, s \in \mathbb{R} .
\end{aligned}
$$

Then,

$$
\begin{aligned}
& B^{\mathbb{Q}}(t)=B(t)-\int_{a}^{t} \phi(\tau) d \tau, \quad a \leq t \leq T, \\
& \tilde{N}^{\mathbb{Q}}(t, A)=N(t, A)-\int_{a}^{t} \int_{\mathbb{R}}(1+\kappa(\tau, s)) Q(\tau, d s) \eta(\tau) d \tau, \quad a \leq t \leq T, \quad A \in \mathfrak{B}(\mathbb{R}),
\end{aligned}
$$

are $(\mathbb{Q}, \mathcal{F})$-Brownian motion and $a(\mathbb{Q}, \mathcal{F})$-random measure. 
Lemma 4. $($ see $[22](p .42))$ Let $\gamma>0$ and $x_{1}, x_{2} \in \mathbb{R}$. Then,

$$
2\left|x_{1} x_{2}\right| \leq \frac{1}{\gamma}\left|x_{1}\right|^{2}+\gamma\left|x_{2}\right|^{2}
$$

Lemma 5. Consider the following family of conformable backward stochastic differential equation parameterized by $n=1,2, \cdots$

$$
\begin{aligned}
X^{n}(t)= & \zeta+\int_{t}^{T}(\tau-a)^{\rho-1} g\left(\tau, X^{n}(\tau), Y^{n}(\tau), Z^{n}(\tau)\right) d \tau+n \int_{t}^{T}(\tau-a)^{\rho-1}\left(X(\tau)-X^{n}(\tau)\right) \\
& -\int_{t}^{T}(\tau-a)^{\rho-1} Y^{n}(\tau) d B(\tau)-\int_{t}^{T}(\tau-a)^{\rho-1} \int_{\mathbb{R}} Z^{n}(\tau, s) \tilde{N}(d \tau, d s), t \in[a, T], 0<\rho \leq 1,
\end{aligned}
$$

where $\zeta \in \mathbb{L}^{2}\left(\Omega, \mathcal{F}_{T}, \mathbb{P}\right), X$ is an adapted process, $Y$ and $Z$ are given control processes, $g$ : $\Omega \times[a, T] \times \mathbb{R} \times \mathbb{R} \times L_{Q}^{2}(\mathbb{R}) \rightarrow \mathbb{R}$ is predictable, $B(\cdot)$ is a given Brownian motion and $\tilde{N}$ is $a$ compensated random measure. For any $n=1,2, \cdots$, we have $X(t) \geq X^{n}(t)$.

Proof. Following [23] (Lemma 3.4), we assume that $X(t)<X^{n}(t)$. Then there exists $\omega>0$ such that the measure of $\left\{(\omega, t): X^{n}(t)-X(t)-\omega \geq 0\right\} \subset \Omega \times[a, T]$ non-zero. Define the following two stopping times:

$$
\begin{aligned}
& \bar{\sigma}=\min \left[T, \inf \left\{t: X^{n}(t) \geq X(t)+\omega\right\}\right] \\
& \bar{\tau}=\inf \left\{t \geq \sigma: X^{n}(t) \leq X(t)\right\}
\end{aligned}
$$

Then we get $\bar{\sigma} \leq \bar{\tau} \leq T$. Since $X(t)-X^{n}(t)$ is right continuous, we have:

$$
\begin{gathered}
X^{n}(\bar{\sigma}) \geq X(\bar{\sigma})+\omega, \\
X^{n}(\bar{\tau}) \leq X(\bar{\tau}) .
\end{gathered}
$$

Suppose $\bar{X}(t)$ is the solution with the terminal value $\bar{X}(\bar{\tau})=X^{n}(\bar{\tau})$ on $[a, \bar{\tau}]$. From (2) and the comparison theorem, we get $X^{n}(\bar{\sigma}) \leq X(\bar{\sigma})$. This is a contradiction. Thus, $X(t) \geq$ $X^{n}(t)$.

\section{The Main Results of $g$-Expectations}

Consider the following conformable backward stochastic differential equation

$$
\begin{aligned}
X(t)= & \zeta+\int_{t}^{T}(\tau-a)^{\rho-1} g(\tau, X(\tau), Y(\tau), Z(\tau, s)) d \tau-\int_{t}^{T}(\tau-a)^{\rho-1} Y(\tau) d B(\tau) \\
& -\int_{t}^{T}(\tau-a)^{\rho-1} \int_{\mathbb{R}} Z(\tau, s) \tilde{N}(d \tau, d s), 0<\rho \leq 1, a \leq t \leq T
\end{aligned}
$$

where $\zeta \in \mathbb{L}^{2}\left(\Omega, \mathcal{F}_{T}, \mathbb{P}\right), X$ is an adapted process, $Y$ and $Z$ are given control processes, $g: \Omega \times[a, T] \times \mathbb{R} \times \mathbb{R} \times L_{Q}^{2}(\mathbb{R}) \rightarrow \mathbb{R}$ is predictable, $B(\cdot)$ is a given Brownian motion and $\tilde{N}$ is a compensated random measure.

Assumption 1. (i) The generator $g: \Omega \times[a, T] \times \mathbb{R} \times \mathbb{R} \times \mathbb{R} \rightarrow \mathbb{R}$ is predictable and Lipschitz in $x$ and $y$

$$
\left|g(t, x, y, z)-g\left(t, x^{\prime}, y^{\prime}, z\right)\right| \leq K\left(\left|x-x^{\prime}\right|+\left|y-y^{\prime}\right|\right), \forall x, x^{\prime}, y, y^{\prime} \in \mathbb{R},
$$

where $K$ is a positive constant.

(ii) For any $z, z^{\prime} \in \mathbb{R}$, there exist constants $-1<C_{1} \leq 0$ and $C_{2} \geq 0$ such that

$$
g(t, x, y, z)-g\left(t, x, y, z^{\prime}\right) \leq \int_{\mathbb{R}}\left(z(t, s)-z^{\prime}(t, s)\right) \delta^{x, y, z, z^{\prime}}(t, s) Q(t, d s) \eta(t),
$$


where $\delta^{x, y, z, z^{\prime}}(t, s): \Omega \times[a, T] \times \mathbb{R} \rightarrow \mathbb{R}$ is predictable and satisfies $C_{1}(1 \wedge|s|) \leq \delta^{x, y, z, z^{\prime}}(t, s) \leq$ $C_{2}(1 \wedge|s|)$.

(iii) For any $x \in \mathbb{R}, g(t, x, 0,0)=0$.

Notice that the comparison theorems in [21] follow from Definition 1. Hence a nonlinear expectation can be defined by conformable backward stochastic differential equations.

Definition 3. A nonlinear expectation $\mathcal{E}_{g}[\cdot]: \mathbb{L}^{2}\left(\Omega, \mathcal{F}_{T}, \mathbb{P}\right) \rightarrow \mathbb{R}$ is called a g-expectation if the generator $g$ of Equation (3) satisfies Assumption 1 and we define the g-expectation as $\mathcal{E}_{g}[\zeta]=X(a)$, where a triple $(X, Y, Z)$ is a unique solution of Equation (3) and $X(a)$ denotes the initial value of the solution.

Definition 4. A nonlinear expectation $\mathcal{E}_{g}\left[\cdot \mid \mathcal{F}_{t}\right]: \mathbb{L}^{2}\left(\Omega, \mathcal{F}_{T}, \mathbb{P}\right) \rightarrow \mathbb{L}^{2}\left(\Omega, \mathcal{F}_{t}, \mathbb{P}\right)$ is called a conditional g-expectation if for any $a \leq t \leq T$, the generator $g$ of Equation (3) satisfies Assumption 1 and we define the conditional g-expectation as $\mathcal{E}_{g}\left[\zeta \mid \mathcal{F}_{t}\right]=X(t)$, where a triple $(X, Y, Z)$ is a unique solution of Equation (3) and $\zeta \in \mathbb{L}^{2}\left(\Omega, \mathcal{F}_{T}, \mathbb{P}\right)$ denotes the terminal value of the solution.

Proposition 1. We have the following results:

(i) For $a \leq t \leq T, A \in \mathcal{F}_{t}$ and $\zeta \in \mathbb{L}^{2}\left(\Omega, \mathcal{F}_{T}, \mathbb{P}\right), \mathcal{E}_{g}\left[\zeta 1_{A} \mid \mathcal{F}_{t}\right]=\mathcal{E}_{g}\left[\zeta \mid \mathcal{F}_{t}\right] 1_{A}$.

(ii) For any $a \leq s \leq t \leq T$ and $\zeta \in \mathbb{L}^{2}\left(\Omega, \mathcal{F}_{T}, \mathbb{P}\right), \mathcal{E}_{g}\left[\mathcal{E}_{g}\left[\zeta \mid \mathcal{F}_{t}\right] \mid \mathcal{F}_{s}\right]=\mathcal{E}_{g}\left[\zeta \mid \mathcal{F}_{s}\right]$.

Proof. Case (i). Let $A \in \mathcal{F}_{t}$. For any $a \leq t \leq T$ and $0<\rho \leq 1$, consider Equation (3) and

$$
\begin{aligned}
X_{1}(t)= & \zeta 1_{A}+\int_{t}^{T}(\tau-a)^{\rho-1} g\left(\tau, X_{1}(\tau), Y_{1}(\tau), Z_{1}(\tau)\right) d \tau \\
& -\int_{t}^{T}(\tau-a)^{\rho-1} Y_{1}(\tau) d B(\tau)-\int_{t}^{T}(\tau-a)^{\rho-1} \int_{\mathbb{R}} Z_{1}(\tau, s) \tilde{N}(d \tau, d s),
\end{aligned}
$$

where $\zeta \in \mathbb{L}^{2}\left(\Omega, \mathcal{F}_{T}, \mathbb{P}\right)$ and the generator $g$ satisfies Assumption 1 . Multiplying by $1_{A}$ on both sides of (3) we get

$$
\begin{aligned}
X(t) 1_{A}= & \zeta 1_{A}+\int_{t}^{T}(\tau-a)^{\rho-1} 1_{A} g(\tau, X(\tau), Y(\tau), Z(\tau)) d \tau \\
& -\int_{t}^{T}(\tau-a)^{\rho-1} Y(\tau) 1_{A} d B(\tau)-\int_{t}^{T}(\tau-a)^{\rho-1} \int_{\mathbb{R}} Z(\tau, s) 1_{A} \tilde{N}(d \tau, d s),
\end{aligned}
$$

where $a \leq t \leq T$ and $0<\rho \leq 1$. Notice that $g(t, X(t), Y(t), Z(t)) 1_{A}=g\left(t, 1_{A} X(t), 1_{A} Y(t)\right.$, $\left.1_{A} Z(t)\right)$, and then,

$$
\begin{aligned}
X(t) 1_{A}= & \zeta 1_{A}+\int_{t}^{T}(\tau-a)^{\rho-1} g\left(\tau, X(\tau) 1_{A}, Y(\tau) 1_{A}, Z(\tau) 1_{A}\right) d \tau \\
& -\int_{t}^{T}(\tau-a)^{\rho-1} Y(\tau) 1_{A} d B(\tau)-\int_{t}^{T}(\tau-a)^{\rho-1} \int_{\mathbb{R}} Z(\tau, s) 1_{A} \tilde{N}(d \tau, d s) .
\end{aligned}
$$

Let $(\bar{X}(t), \bar{Y}(t), \bar{Z}(t))=\left(X(t) 1_{A}, Y(t) 1_{A}, Z(t) 1_{A}\right)$, and (8) can be written as:

$$
\begin{aligned}
\bar{X}(t)= & \zeta 1_{A}+\int_{t}^{T}(\tau-a)^{\rho-1} g(\tau, \bar{X}(\tau), \bar{Y}(\tau), \bar{Z}(\tau)) d \tau \\
& -\int_{t}^{T}(\tau-a)^{\rho-1} \bar{Y}(\tau) d B(\tau)-\int_{t}^{T}(\tau-a)^{\rho-1} \int_{\mathbb{R}} \bar{Z}(\tau, s) \tilde{N}(d \tau, d s) .
\end{aligned}
$$

By the uniqueness of the conformable backward stochastic differential equation, we get $X_{1}(t)=\bar{X}(t)=X(t) 1_{A}, a \leq t \leq T$. From Definition 4, we have $\mathcal{E}_{g}\left[\zeta 1_{A} \mid \mathcal{F}_{t}\right] X_{1}(t)=$ $X(t) 1_{A}=\mathcal{E}_{g}\left[\zeta \mid \mathcal{F}_{t}\right] 1_{A}$. 
Case (ii). For any $A \in \mathcal{F}_{s}$ and $a \leq s \leq t \leq T$, we have $A \in \mathcal{F}_{t}$. From the result of (i), one has:

$$
\begin{aligned}
\mathcal{E}_{g}\left[\mathcal{E}_{g}\left[\mathcal{E}_{g}\left[\zeta \mid \mathcal{F}_{t}\right] \mid \mathcal{F}_{S}\right] 1_{A}\right] & =\mathcal{E}_{g}\left[\mathcal{E}_{g}\left[\mathcal{E}_{g}\left[\zeta \mid \mathcal{F}_{t}\right] 1_{A} \mid \mathcal{F}_{S}\right]\right] \\
& =\mathcal{E}_{g}\left[\mathcal{E}_{g}\left[\mathcal{E}_{g}\left[\zeta 1_{A} \mid \mathcal{F}_{t}\right] \mid \mathcal{F}_{S}\right]\right] \\
& =\mathcal{E}_{g}\left[\mathcal{E}_{g}\left[\zeta 1_{A} \mid \mathcal{F}_{S}\right]\right] \\
& =\mathcal{E}_{g}\left[\mathcal{E}_{g}\left[\zeta \mid \mathcal{F}_{S}\right] 1_{A}\right]
\end{aligned}
$$

where $\zeta \in \mathbb{L}^{2}\left(\Omega, \mathcal{F}_{T}, \mathbb{P}\right)$. Let $\zeta_{1}=\mathcal{E}_{g}\left[\mathcal{E}_{g}\left[\zeta \mid \mathcal{F}_{t}\right] \mid \mathcal{F}_{S}\right]$ and $\zeta_{2}=\mathcal{E}_{g}\left[\zeta \mid \mathcal{F}_{S}\right]$. If we choose $A=\left\{\zeta_{1} \geq \zeta_{2}\right\} \in \mathcal{F}_{t}$, from Definition $1, \zeta_{1} 1_{A} \geq \zeta_{2} 1_{A}$ and $\mathcal{E}_{g}\left[\zeta_{1} 1_{A}\right]=\mathcal{E}_{g}\left[\zeta_{2} 1_{A}\right]$, we get $\zeta_{1} 1_{A}=\zeta_{2} 1_{A}$. Hence $\zeta_{1} \leq \zeta_{2}$. If we set $A=\left\{\zeta_{1} \leq \zeta_{2}\right\} \in \mathcal{F}_{t}$, we get $\zeta_{1} \geq \zeta_{2}$ in the same way. Hence, we conclude that $\zeta_{1}=\zeta_{2}$, that is, $\mathcal{E}_{g}\left[\mathcal{E}_{g}\left[\zeta \mid \mathcal{F}_{t}\right] \mid \mathcal{F}_{s}\right]=\mathcal{E}_{g}\left[\zeta \mid \mathcal{F}_{s}\right]$.

Theorem 1. The g-expectation is $\mathcal{F}$-consistent expectation.

Proof. Let $A \in \mathcal{F}_{t}$. For any $a \leq t \leq T$ and $0<\rho \leq 1$, consider the following equations:

$$
\begin{aligned}
X_{1}(t)= & \zeta 1_{A}+\int_{t}^{T}(\tau-a)^{\rho-1} g\left(\tau, X_{1}(\tau), Y_{1}(\tau), Z_{1}(\tau)\right) d \tau \\
& -\int_{t}^{T}(\tau-a)^{\rho-1} Y_{1}(\tau) d B(\tau)-\int_{t}^{T}(\tau-a)^{\rho-1} \int_{\mathbb{R}} Z_{1}(\tau, s) \tilde{N}(d \tau, d s), \\
X_{2}(t)= & X_{1}(u) 1_{A}+\int_{t}^{T}(\tau-a)^{\rho-1} g\left(\tau, X_{2}(\tau), Y_{2}(\tau), Z_{2}(\tau)\right) d \tau \\
& -\int_{t}^{T}(\tau-a)^{\rho-1} Y_{2}(\tau) d B(\tau)-\int_{t}^{T}(\tau-a)^{\rho-1} \int_{\mathbb{R}} Z_{2}(\tau, s) \tilde{N}(d \tau, d s),
\end{aligned}
$$

where $\zeta \in \mathbb{L}^{2}\left(\Omega, \mathcal{F}_{T}, \mathbb{P}\right), X_{1}(u)=\mathcal{E}_{g}\left[\zeta 1_{A} \mid \mathcal{F}_{t}\right]$ and the generator $g$ satisfies Assumption 1 . Multiplying by $1_{A}$ on both sides of (7) we get:

$$
\begin{aligned}
X_{2}(t) 1_{A}= & \mathcal{E}_{g}\left[\zeta 1_{A} \mid \mathcal{F}_{t}\right] 1_{A}+\int_{t}^{T}(\tau-a)^{\rho-1} 1_{A} g\left(\tau, X_{2}(\tau), Y_{2}(\tau), Z_{2}(\tau)\right) d \tau \\
& -\int_{t}^{T}(\tau-a)^{\rho-1} Y_{2}(\tau) 1_{A} d B(\tau)-\int_{t}^{T}(\tau-a)^{\rho-1} \int_{\mathbb{R}} Z_{2}(\tau, s) 1_{A} \tilde{N}(d \tau, d s)
\end{aligned}
$$

where $a \leq t \leq T$ and $0<\rho \leq 1$. Notice that $g(t, X(t), Y(t), Z(t)) 1_{A}=g\left(t, 1_{A} X(t), 1_{A} Y(t)\right.$, $\left.1_{A} Z(t)\right)$, and then,

$$
\begin{aligned}
X_{2}(t) 1_{A}= & \mathcal{E}_{g}\left[\zeta 1_{A} \mid \mathcal{F}_{t}\right] 1_{A}+\int_{t}^{T}(\tau-a)^{\rho-1} g\left(\tau, X_{2}(\tau) 1_{A}, Y_{2}(\tau) 1_{A}, Z_{2}(\tau) 1_{A}\right) d \tau \\
& -\int_{t}^{T}(\tau-a)^{\rho-1} \Upsilon_{2}(\tau) 1_{A} d B(\tau)-\int_{t}^{T}(\tau-a)^{\rho-1} \int_{\mathbb{R}} Z_{2}(\tau, s) 1_{A} \tilde{N}(d \tau, d s) .
\end{aligned}
$$

Let $\left(X_{3}(t), Y_{3}(t), Z_{3}(t)\right)=\left(X_{2}(t) 1_{A}, Y_{2}(t) 1_{A}, Z_{2}(t) 1_{A}\right)$, and (8) can be written as:

$$
\begin{aligned}
X_{3}(t)= & \mathcal{E}_{g}\left[\zeta 1_{A} \mid \mathcal{F}_{t}\right] 1_{A}+\int_{t}^{T}(\tau-a)^{\rho-1} g\left(\tau, X_{3}(\tau), Y_{3}(\tau), Z_{3}(\tau)\right) d \tau \\
& -\int_{t}^{T}(\tau-a)^{\rho-1} Y_{3}(\tau) d B(\tau)-\int_{t}^{T}(\tau-a)^{\rho-1} \int_{\mathbb{R}} Z_{3}(\tau, s) \tilde{N}(d \tau, d s) .
\end{aligned}
$$

By the uniqueness of the conformable backward stochastic differential equation, we get $X_{1}(t)=X_{3}(t)=\mathcal{E}_{g}\left[\zeta 1_{A} \mid \mathcal{F}_{t}\right] 1_{A}, a \leq t \leq T$. From Definition 3 and Proposition 1 , we have:

$$
\mathcal{E}_{g}\left[\zeta 1_{A}\right]=X_{1}(a)=X_{3}(a)=\mathcal{E}_{g}\left[\mathcal{E}_{g}\left[\zeta 1_{A} \mid \mathcal{F}_{t}\right] 1_{A}\right]=\mathcal{E}_{g}\left[\mathcal{E}_{g}\left[\zeta \mid \mathcal{F}_{t}\right] 1_{A}\right]
$$


Hence, there exists $\xi=\mathcal{E}_{g}\left[\zeta \mid \mathcal{F}_{t}\right]$ such that $\mathcal{E}_{g}\left[\zeta 1_{A}\right]=\mathcal{E}_{g}\left[\xi 1_{A}\right]$.

Next, we prove the uniqueness of $\xi$. Assume that there exists another random variable $\xi^{\prime}$ such that $\mathcal{E}_{g}\left[\xi 1_{A}\right]=\mathcal{E}_{g}\left[\xi^{\prime} 1_{A}\right]$ and $\xi \neq \xi^{\prime}$. Choose $\xi>\xi^{\prime}$. According to the comparison theorem in [21] and Definition 3, we have $\mathcal{E}_{g}\left[\xi 1_{A}\right]>\mathcal{E}_{g}\left[\xi^{\prime} 1_{A}\right]$, which is contrary to $\mathcal{E}_{g}\left[\xi_{1}\right]=\mathcal{E}_{g}\left[\xi^{\prime} 1_{A}\right]$. On the other hand, if we choose $\xi<\xi^{\prime}$, the result $\mathcal{E}_{g}\left[\xi 1_{A}\right]=\mathcal{E}_{g}\left[\xi^{\prime} 1_{A}\right]$ still does not hold. Hence $\xi=\xi^{\prime}$.

Combining the existence and uniqueness of $\xi$, we conclude that the $g$-expectation is $\mathcal{F}$-consistent expectation. The proof is complete.

Next, we give two kinds of $g$-expectation with the special generators $g_{1}$ and $g_{2}$.

Proposition 2. Let $\phi(\cdot)$ and $\kappa(\cdot)$ be $\mathcal{F}$-predictable processes. For any $\mu_{1} \in \mathbb{R}^{+}$and $-1<C_{1} \leq 0$, we define the following generators:

$$
\begin{aligned}
& g_{1}(t, x, y, z)=\mu_{1}|y|+C_{1} \int_{\mathbb{R}}(1 \wedge|s|)|z(t, s)| Q(t, d s) \eta(t), \\
& g_{2}(t, x, y, z)=-\mu_{1}|y|-C_{1} \int_{\mathbb{R}}(1 \wedge|s|)|z(t, s)| Q(t, d s) \eta(t) .
\end{aligned}
$$

Then $\mathcal{E}_{g_{1}}\left[\zeta \mid \mathcal{F}_{t}\right]=\inf _{\mathbb{Q} \in \mathcal{D}} \mathbb{E}^{\mathbb{Q}}\left[\zeta \mid \mathcal{F}_{t}\right]$ and $\mathcal{E}_{g_{2}}\left[\zeta \mid \mathcal{F}_{t}\right]=\sup _{\mathbb{Q} \in \mathcal{D}} \mathbb{E}^{\mathbb{Q}}\left[\zeta \mid \mathcal{F}_{t}\right]$, where

$$
\begin{array}{r}
\mathcal{D}=\left\{\mathbb{Q} \sim \mathbb{P}, \frac{d \mathbb{Q}}{d \mathbb{P}} \mid \mathcal{F}_{t}=M(t), \frac{d M(t)}{M(t)}=\phi(t) d B(t)+\int_{\mathbb{R}} \kappa(t, s) \tilde{N}(d t, d s),\right. \\
\left.M(a)=1,|\phi(t)| \leq \mu_{1},-1<\kappa(t, s) \leq C_{1}(1 \wedge|s|), a \leq t \leq T\right\} .
\end{array}
$$

Proof. Here we consider the case of $g_{1}(t, x, y, z)=\mu_{1}|y|+C_{1} \int_{\mathbb{R}}(1 \wedge|s|)|z(t, s)| Q(t, d s) \eta(t)$. The proof of $g_{2}(t, x, y, z)=-\mu_{1}|y|-C_{1} \int_{\mathbb{R}}(1 \wedge|s|)|z(t, s)| Q(t, d s) \eta(t)$ is similar. Consider the following equation

$$
\begin{aligned}
X(t)= & \zeta+\int_{t}^{T}(\tau-a)^{\rho-1} g_{1}(\tau, X(\tau), Y(\tau), Z(\tau)) d \tau \\
& -\int_{t}^{T}(\tau-a)^{\rho-1} Y(\tau) d B(\tau)-\int_{t}^{T}(\tau-a)^{\rho-1} \int_{\mathbb{R}} Z(\tau, s) \tilde{N}(d \tau, d s) \\
= & \zeta+\int_{t}^{T} \mu_{1}(\tau-a)^{\rho-1}|Y(\tau)| d \tau+\int_{t}^{T}(\tau-a)^{\rho-1} \int_{\mathbb{R}} C_{1}(1 \wedge|s|)|Z(\tau, s)| Q(\tau, d s) \eta(\tau) d \tau \\
& -\int_{t}^{T}(\tau-a)^{\rho-1} Y(\tau) d B(\tau)-\int_{t}^{T}(\tau-a)^{\rho-1} \int_{\mathbb{R}} Z(\tau, s) \tilde{N}(d \tau, d s),
\end{aligned}
$$

where $a \leq t \leq T, 0<\rho \leq 1, \mu_{1} \in \mathbb{R}^{+}$and $-1<C_{1} \leq 0$. Define

$$
\begin{aligned}
& \frac{d \mathbb{Q}}{d \mathbb{P}} \mid \mathcal{F}_{t}=M(t), M(a)=1, \\
& \frac{d M(t)}{M(t)}=\phi(t) d B(t)+\int_{\mathbb{R}} \kappa(t, s) \tilde{N}(d t, d s),
\end{aligned}
$$

where $|\phi(t)| \leq \mu_{1}$ and $-1<\kappa(t, s) \leq C_{1}(1 \wedge|s|)$. Let $\mathcal{D}=\left\{\mathbb{Q} \sim \mathbb{P}, \frac{d \mathbb{Q}}{d \mathbb{P}} \mid \mathcal{F}_{t}=M(t)\right\}$, and from Lemma 3 , we can get 


$$
\begin{aligned}
X(t)= & \zeta+\int_{t}^{T}(\tau-a)^{\rho-1}\left[\mu_{1}|Y(\tau)|-\phi(\tau) Y(\tau)\right] d \tau \\
& +\int_{t}^{T}(\tau-a)^{\rho-1} \int_{\mathbb{R}}\left[C_{1}(1 \wedge|s|)|Z(\tau, s)|-\kappa(\tau, s) Z(\tau, s)\right] Q(\tau, d s) \eta(\tau) d \tau \\
& -\int_{t}^{T}(\tau-a)^{\rho-1} Y(\tau) d B^{\mathbb{Q}}(\tau)-\int_{t}^{T}(\tau-a)^{\rho-1} \int_{\mathbb{R}} Z(\tau, s) \tilde{N}^{\mathbb{Q}}(d \tau, d s) \\
\geq & \zeta-\int_{t}^{T}(\tau-a)^{\rho-1} Y(\tau) d B^{\mathbb{Q}}(\tau)-\int_{t}^{T}(\tau-a)^{\rho-1} \int_{\mathbb{R}} Z(\tau, s) \tilde{N}^{\mathbb{Q}}(d \tau, d s),
\end{aligned}
$$

where $a \leq t \leq T$ and $0<\rho \leq 1$; note that $\phi(\tau) Y(\tau)=\mu_{1} Y(\tau)>0$ and $\kappa(\tau, s) Z(\tau, s)=$ $C_{1}(1 \wedge|s|) Z(\tau, s)>0$, we have

$$
X(t)=\zeta-\int_{t}^{T}(\tau-a)^{\rho-1} Y(\tau) d B^{\mathbb{Q}}(\tau)-\int_{t}^{T}(\tau-a)^{\rho-1} \int_{\mathbb{R}} Z(\tau, s) \tilde{N}^{\mathbb{Q}}(d \tau, d s) .
$$

Taking the conditional expectation under the probability measure $\mathbb{Q}$, we obtain $X(t)=\inf _{\mathbb{Q} \in \mathcal{D}} \mathbb{E}^{\mathbb{Q}}\left[\zeta \mid \mathcal{F}_{t}\right]$. Notice that Assumption 1 is satisfied for the generator $g_{1}(t, x, y, z)$.

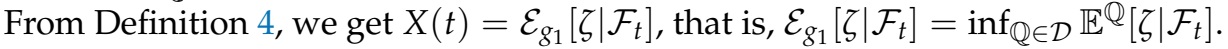

The proof is complete.

Proposition 3. Let $\mathcal{E}_{g}$ be a g-expectation and $\zeta_{,} \zeta_{1}, \zeta_{2} \in \mathbb{L}^{2}\left(\Omega, \mathcal{F}_{T}, \mathbb{P}\right)$.

(i) Translation invariance: for any constant $c \in \mathbb{R}$ and $a \leq t \leq T$, we have

$$
\mathcal{E}_{g}\left[\zeta+c \mid \mathcal{F}_{t}\right]=\mathcal{E}_{g}\left[\zeta \mid \mathcal{F}_{t}\right]+c,
$$

where the generator $g$ is independent of $X(\cdot)$.

(ii) Homogeneity: for any constant $c>0$ and $a \leq t \leq T$, we have

$$
\mathcal{E}_{g}\left[c \zeta \mid \mathcal{F}_{t}\right]=c \mathcal{E}_{g}\left[\zeta \mid \mathcal{F}_{t}\right]
$$

where the generator $g$ is positively homogenous.

(iii) Convexity: for any $c \in(0,1)$ and $a \leq t \leq T$, the g-expectation $\mathcal{E}_{g}$ is convex

$$
\mathcal{E}_{g}\left[c \zeta_{1}+(1-c) \zeta_{2} \mid \mathcal{F}_{t}\right] \leq c \mathcal{E}_{g}\left[\zeta_{1} \mid \mathcal{F}_{t}\right]+(1-c) \mathcal{E}_{g}\left[\zeta_{2} \mid \mathcal{F}_{t}\right]
$$

if the generator $g$ is convex, namely:

$$
\begin{aligned}
& g\left(t, c x_{1}+(1-c) x_{2}, c y_{1}+(1-c) y_{2}, c z_{1}+(1-c) z_{2}\right) \\
\leq \quad & c g\left(t, x_{1}, y_{1}, z_{1}\right)+(1-c) g\left(t, x_{2}, y_{2}, z_{2}\right), \forall\left(x_{1}, y_{1}, z_{1}\right),\left(x_{2}, y_{2}, z_{2}\right) \in\left(\mathbb{R}, \mathbb{R}, L_{Q}^{2}\right) .
\end{aligned}
$$

(iv) Sub-linearity and sub-additivity: the g-expectation $\mathcal{E}_{g}$ is sub-linear, sub-additive and positively homogenous

$$
\mathcal{E}_{g}\left[\zeta_{1}+\zeta_{2} \mid \mathcal{F}_{t}\right] \leq \mathcal{E}_{g}\left[\zeta_{1} \mid \mathcal{F}_{t}\right]+\mathcal{E}_{g}\left[\zeta_{2} \mid \mathcal{F}_{t}\right]
$$

if the generator $g$ is positively homogenous and satisfies:

$$
\begin{aligned}
& g\left(t, x_{1}+x_{2}, y_{1}+y_{2}, z_{1}+z_{2}\right) \\
\leq \quad & g\left(t, x_{1}, y_{1}, z_{1}\right)+g\left(t, x_{2}, y_{2}, z_{2}\right), \forall\left(x_{1}, y_{1}, z_{1}\right),\left(x_{2}, y_{2}, z_{2}\right) \in\left(\mathbb{R}, \mathbb{R}, L_{Q}^{2}\right) .
\end{aligned}
$$


Proof. Case (i). Consider the following conformable backward stochastic differential equations:

$$
\begin{aligned}
X(t)= & \zeta+\int_{t}^{T}(\tau-a)^{\rho-1} g(\tau, X(\tau), Y(\tau), Z(\tau)) d \tau \\
& -\int_{t}^{T}(\tau-a)^{\rho-1} Y(\tau) d B(\tau)-\int_{t}^{T}(\tau-a)^{\rho-1} \int_{\mathbb{R}} Z(\tau, s) \tilde{N}(d \tau, d s), \\
X^{\prime}(t)= & \zeta+c+\int_{t}^{T}(\tau-a)^{\rho-1} g\left(\tau, X^{\prime}(\tau), Y^{\prime}(\tau), Z^{\prime}(\tau)\right) d \tau \\
& -\int_{t}^{T}(\tau-a)^{\rho-1} Y^{\prime}(\tau) d B(\tau)-\int_{t}^{T}(\tau-a)^{\rho-1} \int_{\mathbb{R}} Z^{\prime}(\tau, s) \tilde{N}(d \tau, d s),
\end{aligned}
$$

where $\zeta \in \mathbb{L}^{2}\left(\Omega, \mathcal{F}_{T}, \mathbb{P}\right), c \in \mathbb{R}, a \leq t \leq T$ and $0<\rho \leq 1$. Let

$$
\begin{aligned}
& \frac{d \mathbb{Q}}{d \mathbb{P}} \mid \mathcal{F}_{t}=M(t), M(a)=1 \\
& \frac{d M(t)}{M(t)}=\tilde{\alpha}(t) d B(t)+\int_{\mathbb{R}} \tilde{\beta}(t) \tilde{N}(d t, d s), a \leq t \leq T,
\end{aligned}
$$

where $\tilde{\alpha}(\cdot)$ and $\tilde{\beta}(\cdot)$ are the predictable processes. If we choose a generator $g(t, X(t), Y(t)$, $Z(t))=\tilde{\alpha}(t) Y(t)+\int_{\mathbb{R}} \tilde{\beta}(t) Z(t, s) Q(t, d s) \eta(t)$ which does not depend on $X(\cdot)$, using Lemma 3, one has:

$$
\begin{aligned}
& X(t)=\zeta-\int_{t}^{T}(\tau-a)^{\rho-1} Y(\tau) d B^{\mathbb{Q}}(\tau)-\int_{t}^{T}(\tau-a)^{\rho-1} \int_{\mathbb{R}} Z(\tau, s) \tilde{N}^{\mathbb{Q}}(d \tau, d s), \\
& X^{\prime}(t)=\zeta+c-\int_{t}^{T}(\tau-a)^{\rho-1} Y^{\prime}(\tau) d B^{\mathbb{Q}}(\tau)-\int_{t}^{T}(\tau-a)^{\rho-1} \int_{\mathbb{R}} Z^{\prime}(\tau, s) \tilde{N}^{\mathbb{Q}}(d \tau, d s),
\end{aligned}
$$

where $\zeta \in \mathbb{L}^{2}\left(\Omega, \mathcal{F}_{T}, \mathbb{P}\right), c \in \mathbb{R}, a \leq t \leq T$ and $0<\rho \leq 1$. Hence, we get $X(t)=\mathbb{E}^{\mathbb{Q}}\left[\zeta \mid \mathcal{F}_{t}\right]$ and $X^{\prime}(t)=\mathbb{E}^{\mathbb{Q}}\left[\zeta+c \mid \mathcal{F}_{t}\right]=\mathbb{E}^{\mathbb{Q}}\left[\zeta \mid \mathcal{F}_{t}\right]+c$ under the probability measure $\mathbb{Q}$, that is, $X^{\prime}(t)=$ $X(t)+c, Y(t)=Y^{\prime}(t)$ and $Z(t, s)=Z^{\prime}(t, s)$.

On the other hand, since the generator $g(t, X(t), Y(t), Z(t))=\tilde{\alpha}(t) Y(t)+$ $\int_{\mathbb{R}} \tilde{\beta}(t) Z(t, s) Q(t, d s) \eta(t)$ satisfies Assumption 1, we have $X(t)=\mathcal{E}_{g}\left[\zeta \mid \mathcal{F}_{t}\right]$ and $X^{\prime}(t)=$ $\mathcal{E}_{g}\left[\zeta+c \mid \mathcal{F}_{t}\right]$. Hence, we conclude that $\mathcal{E}_{g}\left[\zeta+c \mid \mathcal{F}_{t}\right]=X^{\prime}(t)=X(t)+c=\mathcal{E}_{g}\left[\zeta \mid \mathcal{F}_{t}\right]+c$.

Case (ii). Let $c>0$, and using the same method as in case (i), consider the following equation:

$$
\begin{aligned}
X^{\prime \prime}(t)= & c \zeta+\int_{t}^{T}(\tau-a)^{\rho-1} g\left(\tau, X^{\prime \prime}(\tau), Y^{\prime \prime}(\tau), Z^{\prime \prime}(\tau)\right) d \tau \\
& -\int_{t}^{T}(\tau-a)^{\rho-1} Y^{\prime \prime}(\tau) d B(\tau)-\int_{t}^{T}(\tau-a)^{\rho-1} \int_{\mathbb{R}} Z^{\prime \prime}(\tau, s) \tilde{N}(d \tau, d s),
\end{aligned}
$$

where $\zeta \in \mathbb{L}^{2}\left(\Omega, \mathcal{F}_{T}, \mathbb{P}\right), a \leq t \leq T$ and $0<\rho \leq 1$. Choose

$$
g\left(t, X^{\prime \prime}(t), Y^{\prime \prime}(t), Z^{\prime \prime}(t)\right)=\tilde{\alpha}(t) Y^{\prime \prime}(t)+\int_{\mathbb{R}} \tilde{\beta}(t) Z^{\prime \prime}(t, s) Q(t, d s) \eta(t),
$$

which is positively homogenous. With the framework of (12), we have

$$
X^{\prime \prime}(t)=c \zeta-\int_{t}^{T}(\tau-a)^{\rho-1} Y^{\prime \prime}(\tau) d B^{\mathbb{Q}}(\tau)-\int_{t}^{T}(\tau-a)^{\rho-1} \int_{\mathbb{R}} Z^{\prime \prime}(\tau, s) \tilde{N}^{\mathbb{Q}}(d \tau, d s),
$$

where $\zeta \in \mathbb{L}^{2}\left(\Omega, \mathcal{F}_{T}, \mathbb{P}\right), a \leq t \leq T$ and $0<\rho \leq 1$. Then we get $X^{\prime \prime}(t)=c \mathbb{E}^{\mathbb{Q}}\left[\zeta \mid \mathcal{F}_{t}\right]$. From Definition 4 and $X(t)=\mathbb{E}^{\mathbb{Q}}\left[\zeta \mid \mathcal{F}_{t}\right]=\mathcal{E}_{g}\left[\zeta \mid \mathcal{F}_{t}\right]$, we have $\mathcal{E}_{g}\left[c \zeta \mid \mathcal{F}_{t}\right]=X^{\prime \prime}(t)=$ $c \mathbb{E}^{\mathbb{Q}}\left[\zeta \mid \mathcal{F}_{t}\right]=c \mathcal{E}_{g}\left[\zeta \mid \mathcal{F}_{t}\right]$. 
Case (iii). Let $0<c<1$, and consider the following conformable backward stochastic differential equations

$$
\begin{aligned}
\bar{X}(t)= & c \zeta_{1}+(1-c) \zeta_{2}+\int_{t}^{T}(\tau-a)^{\rho-1} g(\tau, \bar{X}(\tau), \bar{Y}(\tau), \bar{Z}(\tau)) d \tau \\
& -\int_{t}^{T}(\tau-a)^{\rho-1} \bar{Y}(\tau) d B(\tau)-\int_{t}^{T}(\tau-a)^{\rho-1} \int_{\mathbb{R}} \bar{Z}(\tau, s) \tilde{N}(d \tau, d s), \\
X_{i}(t)= & \zeta_{i}+\int_{t}^{T}(\tau-a)^{\rho-1} g\left(\tau, X_{i}(\tau), Y_{i}(\tau), Z_{i}(\tau)\right) d \tau \\
& -\int_{t}^{T}(\tau-a)^{\rho-1} Y_{i}(\tau) d B(\tau)-\int_{t}^{T}(\tau-a)^{\rho-1} \int_{\mathbb{R}} Z_{i}(\tau, s) \tilde{N}(d \tau, d s),
\end{aligned}
$$

where $i=1,2, \zeta_{i} \in \mathbb{L}^{2}\left(\Omega, \mathcal{F}_{T}, \mathbb{P}\right), 0<c<1, a \leq t \leq T$ and $0<\rho \leq 1$.

Notice that:

$$
\begin{aligned}
& g\left(t, c X_{1}+(1-c) X_{2}, c Y_{1}+(1-c) Y_{2}, c Z_{1}+(1-c) Z_{2}\right) \\
\leq \quad & c g\left(t, X_{1}, Y_{1}, Z_{1}\right)+(1-c) g\left(t, X_{2}, Y_{2}, Z_{2}\right),
\end{aligned}
$$

and we see that:

$$
\begin{aligned}
& g\left(t, c X_{1}+(1-c) X_{2}, c Y_{1}+(1-c) Y_{2}, c Z_{1}+(1-c) Z_{2}\right)+f(t) \\
= & c g\left(t, X_{1}, Y_{1}, Z_{1}\right)+(1-c) g\left(t, X_{2}, Y_{2}, Z_{2}\right),
\end{aligned}
$$

where the nonnegative function $f(t)$ depends on $X_{i}, Y_{i}$ and $Z_{i}$ and $i=1,2$. Let $X_{3}(t)=$ $c X_{1}(t)+(1-c) X_{2}(t), Y_{3}(t)=c Y_{1}(t)+(1-c) Y_{2}(t)$ and $Z_{3}(t)=c Z_{1}(t)+(1-c) Z_{2}(t)$, and we have

$$
\begin{aligned}
X_{3}(t)= & c \zeta_{1}+(1-c) \zeta_{2}+\int_{t}^{T}(\tau-a)^{\rho-1}\left[c g\left(\tau, X_{1}(\tau), Y_{1}(\tau), Z_{1}(\tau)\right)\right. \\
& \left.+(1-c) g\left(\tau, X_{2}(\tau), Y_{2}(\tau), Z_{2}(\tau)\right)\right] d \tau \\
& -\int_{t}^{T}(\tau-a)^{\rho-1}\left[c Y_{1}(\tau)+(1-c) Y_{2}(\tau)\right] d B(\tau) \\
& -\int_{t}^{T}(\tau-a)^{\rho-1} \int_{\mathbb{R}}\left[c Z_{1}(\tau, s)+(1-c) Z_{2}(\tau, s)\right] \tilde{N}(d \tau, d s) \\
= & c \zeta_{1}+(1-c) \zeta_{2}+\int_{t}^{T}(\tau-a)^{\rho-1}\left[g\left(\tau, X_{3}(\tau), Y_{3}(\tau), Z_{3}(\tau)\right)\right. \\
& \left.+f\left(\tau, X_{i}(\tau), Y_{i}(\tau), Z_{i}(\tau)\right)\right] d \tau-\int_{t}^{T}(\tau-a)^{\rho-1} Y_{3}(\tau) d B(\tau) \\
& -\int_{t}^{T}(\tau-a)^{\rho-1} \int_{\mathbb{R}} Z_{3}(\tau, s) \tilde{N}(d \tau, d s),
\end{aligned}
$$

where $i=1,2,0<c<1, \zeta_{i} \in \mathbb{L}^{2}\left(\Omega, \mathcal{F}_{T}, \mathbb{P}\right), a \leq t \leq T$ and $0<\rho \leq 1$. Using the comparison theorem to the Equations (10) and (11), we see that $\bar{X}(t) \leq X_{3}(t)$. From Definition 4 , one has

$\mathcal{E}_{g}\left[c \zeta_{1}+(1-c) \zeta_{2} \mid \mathcal{F}_{t}\right]=\bar{X}(t) \leq X_{3}(t)=c X_{1}(t)+(1-c) X_{2}(t) \leq c \mathcal{E}_{g}\left[\zeta_{1} \mid \mathcal{F}_{t}\right]+(1-c) \mathcal{E}_{g}\left[\zeta_{2} \mid \mathcal{F}_{t}\right]$

where $i=1,2,0<c<1, \zeta_{i} \in \mathbb{L}^{2}\left(\Omega, \mathcal{F}_{T}, \mathbb{P}\right)$ and $a \leq t \leq T$.

Case (iv). Similar to the proof process in case (iii), we get the result in case (iv).

The proof is complete.

Theorem 2. Suppose $g(t, X(t), Y(t), Z(t))=\tilde{\alpha}(t) Y(t)+\int_{\mathbb{R}} \tilde{\beta}(t) Z(t, s) Q(t, d s) \eta(t), a \leq t \leq T$. Then the g-expectation is equivalent to the expectation under a probability measure $\mathbb{Q}$. 
Proof. Consider the following conformable backward stochastic differential equation

$$
\begin{aligned}
X(t)= & \zeta+\int_{t}^{T}(\tau-a)^{\rho-1} g(\tau, X(\tau), Y(\tau), Z(\tau)) d \tau \\
& -\int_{t}^{T}(\tau-a)^{\rho-1} Y(\tau) d B(\tau)-\int_{t}^{T}(\tau-a)^{\rho-1} \int_{\mathbb{R}} Z(\tau, s) \tilde{N}(d \tau, d s),
\end{aligned}
$$

where $\zeta \in \mathbb{L}^{2}\left(\Omega, \mathcal{F}_{T}, \mathbb{P}\right), a \leq t \leq T$ and $0<\rho \leq 1$.

It is clear that the generator $g(t, X(t), Y(t), Z(t))=\tilde{\alpha}(t) Y(t)+\int_{\mathbb{R}} \tilde{\beta}(t) Z(t, s) Q(t, d s) \eta(t)$ satisfies Assumption 1. From Definition 4, we have $X(t)=\mathcal{E}_{g}\left[\zeta \mid \mathcal{F}_{t}\right]$.

Let

$$
\begin{aligned}
& \frac{d \mathbb{Q}}{d \mathbb{P}} \mid \mathcal{F}_{t}=M(t), M(a)=1 \\
& \frac{d M(t)}{M(t)}=\tilde{\alpha}(t) d B(t)+\int_{\mathbb{R}} \tilde{\beta}(t) \tilde{N}(d t, d s), a \leq t \leq T,
\end{aligned}
$$

where $\tilde{\alpha}(\cdot)$ and $\tilde{\beta}(\cdot)$ are the predictable processes. Using Lemma 3, one has:

$$
X(t)=\zeta-\int_{t}^{T}(\tau-a)^{\rho-1} Y(\tau) d B^{\mathbb{Q}}(\tau)-\int_{t}^{T}(\tau-a)^{\rho-1} \int_{\mathbb{R}} Z(\tau, s) \tilde{N}^{\mathbb{Q}}(d \tau, d s),
$$

where $\zeta \in \mathbb{L}^{2}\left(\Omega, \mathcal{F}_{T}, \mathbb{P}\right), a \leq t \leq T$ and $0<\rho \leq 1$. Hence, we get $X(t)=\mathbb{E}^{\mathbb{Q}}\left[\zeta \mid \mathcal{F}_{t}\right]$ under the probability measure $\mathbb{Q}$. From the uniqueness of the solution, we conclude that $\mathcal{E}_{g}\left[\zeta \mid \mathcal{F}_{t}\right]=X(t)=\mathbb{E}^{\mathbb{Q}}\left[\zeta \mid \mathcal{F}_{t}\right]$. The proof is complete.

\section{Doob-Meyer Decomposition Theorem}

We first give some definitions.

Definition 5. The process $X(\cdot)$ is called a g-martingale if for any $a \leq s \leq t \leq T$, we have $\mathbb{E}\left[|X(t)|^{2}\right]<\infty$ and

$$
\mathcal{E}_{g}\left[X(t) \mid \mathcal{F}_{s}\right]=X(s)
$$

Definition 6. The process $X(\cdot)$ is called a g-supermartingale if for any $a \leq s \leq t \leq T$, we have $\mathbb{E}\left[|X(t)|^{2}\right]<\infty$ and

$$
\mathcal{E}_{g}\left[X(t) \mid \mathcal{F}_{s}\right] \leq X(s)
$$

Theorem 3. Assume that the generator $g$ satisfies Assumption 1. If the process $X(\cdot) \in \mathbb{S}^{2}(\mathbb{R})$ is a g-supermartingale on $[a, T]$, then there exists a unique triple $(Y, Z) \in \mathbb{H}^{2}(\mathbb{R}) \times \mathbb{H}_{N}^{2}(\mathbb{R})$ and a continuous increasing process $A(\cdot)$ such that:

$$
\begin{aligned}
X(t)= & \zeta+\int_{t}^{T}(\tau-a)^{\rho-1} g(\tau, X(\tau), Y(\tau), Z(\tau)) d \tau+A(T)-A(t) \\
& -\int_{t}^{T}(\tau-a)^{\rho-1} Y(\tau) d B(\tau)-\int_{t}^{T}(\tau-a)^{\rho-1} \int_{\mathbb{R}} Z(\tau, s) \tilde{N}(d \tau, d s),
\end{aligned}
$$

where $\zeta \in \mathbb{L}^{2}\left(\Omega, \mathcal{F}_{T}, \mathbb{P}\right), a \leq t \leq T, 0<\rho \leq 1, A(a)=0$ and $\mathbb{E}\left[|A(T)|^{2}\right]<\infty$.

Proof. Consider the following conformable backward stochastic differential equation:

$$
\begin{aligned}
X^{n}(t)= & \zeta+\int_{t}^{T}(\tau-a)^{\rho-1} g^{n}\left(\tau, X^{n}(\tau), Y^{n}(\tau), Z^{n}(\tau)\right) d \tau \\
& -\int_{t}^{T}(\tau-a)^{\rho-1} Y^{n}(\tau) d B(\tau)-\int_{t}^{T}(\tau-a)^{\rho-1} \int_{\mathbb{R}} Z^{n}(\tau, s) \tilde{N}(d \tau, d s),
\end{aligned}
$$

where $\zeta \in \mathbb{L}^{2}\left(\Omega, \mathcal{F}_{T}, \mathbb{P}\right), a \leq t \leq T, 0<\rho \leq 1$ and $n=1,2,3, \cdots$. 
Assume that $g^{n}(t, x, y, z)=g(t, x, y, z)+n(X(t)-x)$, so Equation (13) can be written as:

$$
\begin{aligned}
X^{n}(t)= & \zeta+\int_{t}^{T}(\tau-a)^{\rho-1} g\left(\tau, X^{n}(\tau), Y^{n}(\tau), Z^{n}(\tau)\right) d \tau+A^{n}(T)-A^{n}(t) \\
& -\int_{t}^{T}(\tau-a)^{\rho-1} Y^{n}(\tau) d B(\tau)-\int_{t}^{T}(\tau-a)^{\rho-1} \int_{\mathbb{R}} Z^{n}(\tau, s) \tilde{N}(d \tau, d s),
\end{aligned}
$$

where $A^{n}(t)=\int_{a}^{t} n(\tau-a)^{\rho-1}\left(X(\tau)-X^{n}(\tau)\right) d \tau$. From Lemma 5 and a comparison theorem, we get that the sequence $\left(X^{n}(t)\right)_{n \in \mathbb{N}^{+}}$is increasing and monotonically converges. Hence the sequence $\left(A^{n}(t)\right)_{n \in \mathbb{N}^{+}}$is continuous and increasing. From Equation (14), we have

$$
\begin{aligned}
A^{n}(T)= & X^{n}(a)-\zeta-\int_{a}^{T}(\tau-a)^{\rho-1} g\left(\tau, X^{n}(\tau), Y^{n}(\tau), Z^{n}(\tau)\right) d \tau \\
& +\int_{a}^{T}(\tau-a)^{\rho-1} Y^{n}(\tau) d B(\tau)+\int_{a}^{T}(\tau-a)^{\rho-1} \int_{\mathbb{R}} Z^{n}(\tau, s) \tilde{N}(d \tau, d s) \\
\leq & \left|X^{n}(a)\right|+|\zeta|+k \int_{a}^{T}(\tau-a)^{\rho-1}\left[\left|X^{n}(\tau)\right|+\left|Y^{n}(\tau)\right|+\int_{\mathbb{R}}\left|Z^{n}(\tau, s)\right| Q(\tau, d s) \eta(\tau)\right] d \tau \\
& +\int_{a}^{T}(\tau-a)^{\rho-1}\left|Y^{n}(\tau)\right| d B(\tau)+\int_{a}^{T}(\tau-a)^{\rho-1} \int_{\mathbb{R}}\left|Z^{n}(\tau, s)\right| \tilde{N}(d \tau, d s),
\end{aligned}
$$

where $k$ depends on $K$ and $\delta^{x, y, z, z^{\prime}}(t, s)$. Then,

$$
\begin{aligned}
\mathbb{E}\left[\left|A^{n}(T)\right|^{2}\right] \leq & \mathbb{E}\left[\left|X^{n}(a)\right|^{2}\right]+\mathbb{E}\left[\zeta^{2}\right]+k_{1} \mathbb{E}\left[\sup _{a \leq t \leq T}\left|X^{n}(t)\right|^{2}\right] \\
& +k_{2} \mathbb{E}\left[\int_{a}^{T}(\tau-a)^{2(\rho-1)}\left(\left|Y^{n}(\tau)\right|^{2}+\int_{\mathbb{R}}\left|Z^{n}(\tau, s)\right|^{2} Q(\tau, d s) \eta(\tau)\right) d \tau\right],
\end{aligned}
$$

for a $k_{1}$ depending on $T, \rho$ and $a$, and $k_{2}$ is a constant. Hence, there exists a constant $l_{1}$ such that:

$\mathbb{E}\left[\left|A^{n}(T)\right|^{2}\right] \leq l_{1}+k_{2} \mathbb{E}\left[\int_{a}^{T}(\tau-a)^{2(\rho-1)}\left(\left|Y^{n}(\tau)\right|^{2}+\int_{\mathbb{R}}\left|Z^{n}(\tau, s)\right|^{2} Q(\tau, d s) \eta(\tau)\right) d \tau\right]$,

where $a \leq t \leq T, 0<\rho \leq 1$ and $n=1,2,3, \cdots$. Apply Lemma 2 to $\left|X^{n}(t)\right|^{2}$, and we have:

$$
\begin{aligned}
\left|X^{n}(t)\right|^{2}= & \zeta^{2}+2 \int_{t}^{T}(\tau-a)^{\rho-1}\left|X^{n}(\tau)\right| g\left(\tau, X^{n}(\tau), Y^{n}(\tau), Z^{n}(\tau)\right) d \tau \\
& +2 \int_{t}^{T}\left|X^{n}(\tau)\right| d A^{n}(\tau)-2 \int_{t}^{T}(\tau-a)^{\rho-1}\left|X^{n}(\tau)\right| Y^{n}(\tau) d B(\tau) \\
& -2 \int_{t}^{T}(\tau-a)^{\rho-1}\left|X^{n}(\tau)\right| \int_{\mathbb{R}} Z^{n}(\tau, s) \tilde{N}(d \tau, d s)-\int_{t}^{T}\left(d X^{n}(\tau)\right)^{2},
\end{aligned}
$$

where $a \leq t \leq T, 0<\rho \leq 1$ and $n=1,2,3, \cdots$. According to Lemma 4, Assumption 1 and Lemma 1, one has: 


$$
\begin{aligned}
& \mathbb{E}\left[\left|X^{n}(t)\right|^{2}\right] \leq \mathbb{E}\left[\zeta^{2}\right]+2 \mathbb{E}\left[\int_{t}^{T}(\tau-a)^{\rho-1}\left|X^{n}(\tau)\right| g\left(\tau, X^{n}(\tau), Y^{n}(\tau), Z^{n}(\tau)\right) d \tau\right] \\
&+2 \mathbb{E}\left[\int_{t}^{T}\left|X^{n}(\tau)\right| d A^{n}(\tau)\right]-\mathbb{E}\left[\int_{t}^{T}(\tau-a)^{2(\rho-1)}\left|Y^{n}(\tau)\right|^{2} d \tau\right] \\
&-\mathbb{E}\left[\int_{t}^{T} \int_{\mathbb{R}}(\tau-a)^{2(\rho-1)}\left|Z^{n}(\tau, s)\right|^{2} Q(\tau, d s) \eta(\tau) d \tau\right] \\
& \leq \mathbb{E}\left[\zeta^{2}\right]+2 K \mathbb{E}\left[\int_{t}^{T}(\tau-a)^{\rho-1}\left|X^{n}(\tau)\right|^{2} d \tau\right]+2 K \mathbb{E}\left[\int_{t}^{T}(\tau-a)^{\rho-1}\left|X^{n}(\tau)\right| Y^{n}(\tau) d \tau\right] \\
&+2 k \mathbb{E}\left[\int_{t}^{T} \int_{\mathbb{R}}(\tau-a)^{\rho-1}\left|X^{n}(\tau)\right| Z^{n}(\tau, s) Q(\tau, d s) \eta(\tau) d \tau\right]+2 \mathbb{E}\left[\int_{t}^{T}\left|X^{n}(\tau)\right| d A^{n}(\tau)\right] \\
&-\mathbb{E}\left[\int_{t}^{T}(\tau-a)^{2(\rho-1)}\left|Y^{n}(\tau)\right|^{2} d \tau\right]-\mathbb{E}\left[\int_{t}^{T} \int_{\mathbb{R}}(\tau-a)^{2(\rho-1)}\left|Z^{n}(\tau, s)\right|^{2} Q(\tau, d s) \eta(\tau) d \tau\right] \\
& \leq \mathbb{E}\left[\zeta^{2}\right]+2 K \mathbb{E}\left[\int_{t}^{T}(\tau-a)^{\rho-1}\left|X^{n}(\tau)\right|^{2} d \tau\right]+(\gamma K-1) \mathbb{E}\left[\int_{t}^{T}(\tau-a)^{2(\rho-1)}\left|Y^{n}(\tau)\right|^{2} d \tau\right] \\
&+\frac{1}{\gamma}(K+k+1) \mathbb{E}\left[\int_{t}^{T}\left|X^{n}(\tau)\right|^{2} d \tau\right]+\gamma \mathbb{E}\left[\left|A^{n}(T)\right|^{2}\right] \\
&+(\gamma K-1) \mathbb{E}\left[\int_{t}^{T} \int_{\mathbb{R}}(\tau-a)^{2(\rho-1)}\left|Z^{n}(\tau, s)\right|^{2} Q(\tau, d s) \eta(\tau) d \tau\right], \\
& \text { namely, } \quad \mathbb{E}^{\leq}\left[\int_{a}^{T}(\tau-a)^{2(\rho-1)}\left(\left|Y^{n}(\tau)\right|^{2}+\int_{\mathbb{R}}\left|Z^{n}(\tau, s)\right|^{2} Q(\tau, d s) \eta(\tau)\right) d \tau\right] \\
& \leq l_{2}+\frac{\gamma}{1-\gamma K} \mathbb{E}\left[\left|A^{n}(T)\right|^{2}\right],
\end{aligned}
$$

where $l_{2}$ is a constant and $\gamma \leq \frac{1}{R}$. Combining Equations (15) and (16), we conclude that there exists a constant $C$, independent of $n$, such that $\mathbb{E}\left[\left|A^{n}(T)\right|^{2}\right] \leq C$ and

$$
\mathbb{E}\left[\int_{a}^{T}(\tau-a)^{2(\rho-1)}\left|Y^{n}(\tau)\right|^{2} d \tau\right]+\mathbb{E}\left[\int_{a}^{T} \int_{\mathbb{R}}(\tau-a)^{2(\rho-1)}\left|Z^{n}(\tau, s)\right|^{2} Q(\tau, d s) \eta(\tau) d \tau\right] \leq C,
$$

where $a \leq t \leq T, 0<\rho \leq 1$ and $n=1,2,3, \cdots$. In addition, we also get

$$
\mathbb{E}\left[\int_{a}^{T}(\tau-a)^{2(\rho-1)} g\left(\tau, X^{n}(\tau), Y^{n}(\tau), Z^{n}(\tau)\right) d \tau\right] \leq C .
$$

In other words, these sequences $\left(X^{n}(t)\right)_{n \in \mathbb{N}^{+}},\left(Y^{n}(t)\right)_{n \in \mathbb{N}^{+}}$and $\left(Z^{n}(t, \cdot)\right)_{n \in \mathbb{N}^{+}}$weakly converge in their spaces, and then for all stopping time $\zeta$, we have

$$
\begin{gathered}
\lim _{n \rightarrow \infty} \int_{a}^{\varsigma}(\tau-a)^{\rho-1} X^{n}(\tau) d \tau=\int_{a}^{\varsigma}(\tau-a)^{\rho-1} X(\tau) d \tau, \\
\lim _{n \rightarrow \infty} \int_{a}^{\varsigma}(\tau-a)^{\rho-1} Y^{n}(\tau) d B(\tau)=\int_{a}^{\varsigma}(\tau-a)^{\rho-1} Y(\tau) d B(\tau),
\end{gathered}
$$

and

$$
\lim _{n \rightarrow \infty} \int_{a}^{\varsigma}(\tau-a)^{\rho-1} \int_{\mathbb{R}} Z^{n}(\tau, s) \tilde{N}(d \tau, d s)=\int_{a}^{\varsigma}(\tau-a)^{\rho-1} \int_{\mathbb{R}} Z(\tau, s) \tilde{N}(d \tau, d s),
$$


and hence we have:

$$
\begin{aligned}
X(t)= & \zeta+\int_{t}^{T}(\tau-a)^{\rho-1} g(\tau, X(\tau), Y(\tau), Z(\tau)) d \tau+A(T)-A(t) \\
& -\int_{t}^{T}(\tau-a)^{\rho-1} Y(\tau) d B(\tau)-\int_{t}^{T}(\tau-a)^{\rho-1} \int_{\mathbb{R}} Z(\tau, s) \tilde{N}(d \tau, d s),
\end{aligned}
$$

where a continuous increasing process $A(\cdot)$ satisfies $A(a)=0$ and $\mathbb{E}\left[|A(T)|^{2}\right]<\infty$.

Author Contributions: The contributions of all authors (M.L., M.F., J.-R.W. and D.O.) are equal. All the main results were developed together. All authors have read and agreed to the published version of the manuscript.

Funding: This work is partially supported by the Training Object of High Level and Innovative Talents of Guizhou Province ((2016)4006), Major Research Project of Innovative Group in Guizhou Education Department ([2018]012), Guizhou Data Driven Modeling Learning and Optimization Innovation Team ([2020]5016), the Slovak Research and Development Agency under the contract No. APVV-18-0308, and the Slovak Grant Agency VEGA No. 1/0358/20 and No. 2/0127/20.

Institutional Review Board Statement: Not applicable.

Informed Consent Statement: Not applicable.

Data Availability Statement: Not applicable.

Acknowledgments: The authors are grateful to the referees for their careful reading of the manuscript and valuable comments. The authors thank the editor too.

Conflicts of Interest: The authors declare no conflict of interest.

\section{References}

1. Choquet, G. Theory of capacities. Ann. L'Institut Fourier 1954, 5, 131-295. [CrossRef]

2. Coquet, F.; Hu, Y.; Mémin, J.; Peng, S.G. Filtration-consistent nonlinear expectations and related g-expectations. Probab. Theory Relat. Fields 2002, 123, 1-27. [CrossRef]

3. Peng, S.G. Nonlinear Expectations, Nonlinear Evaluations and Risk Measures. In Stochastic Methods in Finance. Lecture Notes in Mathematics; Springer: Berlin/Heidelberg, Germany, 2004; pp. 165-253.

4. Royer, M. Backward stochastic differential equations with jumps and related non-linear expectations. Stoch. Process. Their Appl. 2006, 116, 1358-1376. [CrossRef]

5. Li, H.W.; Peng, S.G. Reflected backward stochastic differential equation driven by G-Brownian motion with an upper obstacle. Stoch. Process. Their Appl. 2020, 130, 6556-6579. [CrossRef]

6. Zheng, S.Q.; Zhang, L.D.; Feng, L.C. On the backward stochastic differential equation with generator $f(y)|z|^{2}$. J. Math. Anal. Appl. 2021, 500, 125102. [CrossRef]

7. Nam, K. Locally Lipschitz BSDE driven by a continuous martingale a path-derivative approach. Stoch. Process. Their Appl. 2021, 141, 376-411. [CrossRef]

8. Moon, J. The risk-sensitive maximum principle for controlled forward-backward stochastic differential equations. Automatica 2020, 120, 10906. [CrossRef]

9. Tangpi, L. Concentration of dynamic risk measures in a Brownian filtration. Stoch. Process. Their Appl. 2018, 129, 1477-1491. [CrossRef]

10. Long, T.; Lapitckii, A.; Guenther, M. A multi-step scheme based on cubic spline for solving backward stochastic differential equations. Appl. Numer. Math. 2020, 150, 117-138.

11. Chen, X.; Ye, W.J. A study of backward stochastic differential equation on a Riemannian manifold. Electron. J. Probab. 2021, 26, 1-31. [CrossRef]

12. Jing, Y.Y.; Li, Z. Averaging principle for backward stochastic differential equations. Discret. Dyn. Nat. Soc. 2021, 2, 1-10. [CrossRef]

13. Zheng, G.Q. Local wellposedness of coupled backward stochastic differential equations driven by G-Brownian motions. J. Math Anal. Appl. 2022, 506, 125540. [CrossRef]

14. Luo, P.; Olivier, M.P.; Ludovic, T.P. Strong solutions of forward-backward stochastic differential equations with measurable coefficients. Stoch. Process. Their Appl. 2022, 144, 1-22. [CrossRef]

15. Qiu, W.Z.; Fečkan, M.; O'Regan, D.; Wang, J. Convergence analysis for iterative learning control of conformable impulsive differential equations. Bull. Iran. Math. Soc. 2022, 48, 193-212. [CrossRef]

16. Ding, Y.L.; O'Regan, D.; Wang, J. Stability analysis for conformable non-instantaneous impulsive differential equations. Bull. Iran. Math. Soc. 2021. [CrossRef] 
17. Ding, Y.L.; Fečkan, M.; Wang, J. Conformable linear and nonlinear non-instantaneous impulsive differential equations. Electron. J. Differ. Equ. 2020, 2020, 1-19.

18. Li, M.M.; Wang, J.; O'Regan, D. Existence and Ulam's stability for conformable fractional differential equations with constant coefficients. Bull. Malays. Math. 2019, 40, 1791-1812. [CrossRef]

19. Qiu, W.Z.; Wang, J.; O'Regan, D. Existence and Ulam stability of solutions for conformable impulsive differential equations. Bull. Iran. Math. Soc. 2020, 46, 1613-1637. [CrossRef]

20. Xiao, G.L.; Wang, J.; O'Regan, D. Existence and stability of solutions to neutral conformable stochastic functional differential equations. Qual. Theory Dyn. Syst. 2022, 21, 7. [CrossRef]

21. Luo, M.; Wang, J.; O'Regan, D. A class of conformable backward stochastic differential equations with jumps. Miskolc Math. Notes 2021, code: MMN-3766.

22. Delong, Ł. Backward Stochastic Differential Equations with Jumps and Their Actuarial and Financial Applications; Springer: London, UK, 2013; 288p.

23. Peng, S.G. Monotonic limit theorem of BSDE and nonlinear decomposition theorem of Doob-Meyer's type. Probab. Theorem Relat. Fields 1999, 113, 473-499. [CrossRef] 